\title{
La deserción en los posgrados, un problema no menor
}

DOI: https://doi.org/10.32870/dse.v0i8.309

\section{Omar Karim Hernández Romo* Ricardo Pérez Mora** Guillermo González Estévez***}

\begin{abstract}
Resumen: Las organizaciones de educación públicas o privadas, en los diferentes niveles escolares, se encuentran en la continua búsqueda de datos primarios que sirvan para la formulación de estrategias que ayuden a mejorar la calidad de su razón de ser; en este entendido, las instituciones, los dirigentes educativos e investigadores han enfocado sus esfuerzos en los estudios de trayectorias escolares, laborales, seguimiento a egresados, entre otros, en busca de mejorar la eficiencia terminal.

Sin embargo, se carece de investigaciones que identifiquen, diagnostiquen y analicen las principales características, causas, perspectivas, motivaciones e implicaciones de la deserción estudiantil. Páramo y Correa (1999: 71) sostienen que "la deserción es, por excelencia, un problema del sistema educativo, íntimamente ligado a los entornos, contornos y dintornos del mismo, tales como los ambientes educativos, situaciones familiares, exigencias ambientales y culturales que afectan directamente al desertor", en este entendido Osorio y Jaramillo consideran que "la deserción aparece como un indicador de crisis del sistema educativo, por cuanto la eficiencia de tal sistema debiera permitir la retención de sus estudiantes y permitirles cursar sus actividades curriculares" (Osorio y Jaramillo, 1999: 1). "En México, el incremento de la deserción en nivel medio superior de acuerdo con la Organización para la Cooperación y el Desarrollo Económico (OCDE), está asociado a un bajo presupuesto para la educación, sumado al bajo ingreso percibido por sus habitantes" (OCDE, 2004). En este contexto, el presente artículo analiza las características que envuelven a los ex alumnos que desertan de los programas de posgrado; para exponer lo investigado, primero se aborda el tema de la deserción, los factores que intervienen en ella y posteriormente el caso de los posgrados del Centro Universitario de Ciencias Económico Administrativas de la Universidad de Guadalajara. Palabras clave: deserción, posgrados, CUCEA, Universidad de Guadalajara (UdG).
\end{abstract}

\begin{abstract}
Studies where analysis case are students or alumni have emerged several years, principally by the need to improve institutions and entities requirements such as CONACYT evaluators, institution accredited graduate degree programs through a quality standards through compliance indicators, including one of the most important is the terminal efficiency, which means in many cases the culmination of a personal and institutional project, nevertheless, the main research has focused on student trajectories, work or follow up of graduates, nonetheless, studies of alumni who interrupt their school projects are poorly developed and considering that these cases referred directly impact indicator, should be promoted more intensity. The present investigation was vested as main objective to analyze the characteristics that involve alumni who drop out of graduate programs at the University Center of Economic and Administrative Sciences (CUCEA) at the University of Guadalajara (U. de G.), as input to strategy formulation and decision -making in pursuit of continuous improvement. This, of that education programs at the graduate level, require continuous adjustments to reflect present and future changes, on the other hand the performance of public and private
\end{abstract}

* Secretario de la Coordinación General de Planeación y Desarrollo Institucional de la Universidad de Guadalajara. Pedro Moreno núm. 966, Col. Centro C.P. 44100, Guadalajara. Correo electrónico: omar.karim@redudg.udg.mx

**Jefe del Departamento de Políticas Públicas del CUCEA. Periférico Norte núm. 799, Núcleo Universitario Los Belenes, C.P. 45100, Zapopan. Correo electrónico: r_pm2001@yahoo.com

**Analista especializado PIFI de la Coordinación General de Planeación y Desarrollo Institucional de la Universidad de Guadalajara. Pedro Moreno núm. 966, Col. Centro C.P. 44100, Guadalajara. Correo electrónico: memo016@hotmail.com 
organizational objectives are demanding constant updates on the processes related to the use of their resources and continuous improvement of its action. Finally, the study was conducted under a research context with qualitative and quantitative approach, using a methodology exploratory and descriptive, using a non-experimental research design, cross-sectional survey using the technique, the results were processed using the SPSS software, to finally analyze the findings, determine the characteristics and findings of the investigation in question. Key words: reasons, drop out, postgraduate, CUCEA, University of Guadalajara.

\section{La deserción estudiantil}

La educación es considerada como el factor crucial en las economías de los países. El sistema educativo, que cada país adopte, debe de estar diseñado para cubrir las necesidades de desarrollo y aspiraciones de la nación. En otras palabras la competitividad se logra mediante la educación de la sociedad, principalmente la educación superior y el posgrado, como el grado de especialización de mayor impacto. La educación superior pública es vista como un sistema planeado, organizado, estructurado y dirigido por el Estado, reconocido como un bien público, a través del cual se forman a las personas para una vida social y laboral, dicha tarea debe responder a indicadores de calidad que aseguren en la medida de lo posible, los macro objetivos que se plantean en el sector, que abonen al desarrollo de la nación y que sean reconocidos por la sociedad.

Cuando se habla de calidad en la educación se considera como uno de los principales indicadores a la eficiencia terminal, misma que es entendida como el número de alumnos que ingresa a un programa académico, es el mismo número que debe de concluir y además de certificar sus estudios (Zúñiga, 2006).

Otros indicadores que se utilizan para evaluar la calidad del sistema educativo son: la cobertura, el índice de absorción, el índice de reprobación, el rezago escolar, el grado académico de los profesores, la productividad académica, el currículo institucional, las metodologías de autoevaluación, la infraestructura, entre otros. Sin embargo, el indicador por excelencia es la eficiencia terminal, la cual está ligada directamente con la deserción.

A pesar de la gran cantidad de estudios que se han realizado a nivel nacional e internacional sobre el fenómeno de la deserción, principalmente en la educación básica, media superior, superior y algunas cuantas de posgrado, al hablar del significado se ha encontrado que existen múltiples definiciones. Esto sucede principalmente porque los investigadores en el área, como menciona Muñoz, no cuentan con un criterio único que les permita estudiar con precisión a los desertores (Muñoz et al., 1979).

Vincent Tinto recomienda que cada institución marque el entendido de este concepto para que sea definido en función del estudio (1987), el mismo autor y uno de los pioneros del estudio del fenómeno, define como desertor a todo alumno que abandona las instituciones de educación superior.

Igualmente, entre las primeras definiciones de deserción se encuentra la expresada por Páramo y Correa (1999: 67), quienes indican que la deserción debe entenderse como el "abandono definitivo de las aulas de clase por diferentes razones y la no continuidad en la formación académica que la sociedad quiere y desea para cada persona que inicia sus estudios de primaria, esperanzados de terminar felizmente sus estudios universitarios". 
En palabras de Rumberger (2001), la deserción incluye a las personas que no concluyen un nivel de estudios y no están inscritos en escuelas que les permitan terminarlos en determinado tiempo. Para Osorio y Jaramillo "la deserción aparece como un indicador de crisis del sistema educativo, por cuanto la eficiencia de tal sistema debiera permitir la retención de sus estudiantes y permitirles cursar sus actividades curriculares" (1999: 1).

Desde un carácter más institucional, la Secretaría de Educación Pública define la deserción como "el abandono de las actividades escolares antes de terminar algún grado o nivel educativo" (SEP, 2004). Con base en lo antes mencionado, la presente investigación entiende la deserción como: la interrupción que hacen los alumnos de los estudios emprendidos antes de culminar el total de las asignaturas determinadas por el programa de académico en el cual estaban matriculados.

Si bien las múltiples definiciones de la deserción otorgan un panorama de la complejidad del fenómeno, es interesante atender los expuestos por Arboleda y Picón (1997: 20), cuando introduce el concepto "episodio de deserción como la unidad de medida del fenómeno, según la cual un estudiante puede abandonar en varias oportunidades un mismo programa, de manera forzosa $\mathrm{o}$ voluntaria".

Al momento de conceptualizar la deserción en la educación superior, Páramo y Correa (1999: 68) han clasificado dicho fenómeno en:

1. Deserción total: abandono definitivo de la formación académica individual.

2. Deserción discriminada por causas: según la causa de la deserción.

3. Deserción por facultad: cambio de institución.

4. Deserción en primer semestre de carrera: por inadecuada adaptación a la vida universitaria.

5. Deserción acumulada: sumatoria de deserciones en una institución.

Por su parte Magallón, Calderón y Soto (2006: 11) clasifican la deserción, en dos tipologías, la primera con base en el tiempo:

1. Inicial: se refiere a los estudiantes que interrumpen sus estudios en el primer semestre del programa académico.

2. Temprana: alude a los desertores de segundo y quinto semestre de estudios.

3. Tardía: cuando los alumnos desertan del sexto semestre en adelante.

La segunda con base al espacio:

1. Interna: los estudiantes que deciden cursar otro programa de estudios en una misma institución. 
2. Institucional: hace referencia al estudiante que abandona definitivamente una institución educativa.

3. Del sistema: es cuando los ex alumnos de una institución no se inscriben en ninguna otra para continuar sus estudios.

\section{Factores que intervienen en de la deserción}

Los estudios de deserción han generado múltiples conceptos, y más aún factores que originan la salida de los estudiantes de sus programas de estudio e instituciones educativas, diferentes autores aluden a la existencia de factores personales y contextuales que tiene un impacto diferenciado en dicho fenómeno, de los primeros Kember (1989), incluye un análisis costo-beneficio cuando el estudiante valora el costo de oportunidad del tiempo dedicado a estudiar y el beneficio que percibirá al culminar su plan de estudios; en este sentido, Hackman y Dysinger (1970), consideran que algunos estudiantes no están suficientemente comprometidos con la graduación universitaria, en estos casos la deserción es el resultado bajo niveles de interés.

Respecto a los factores contextuales se encuentran los sociales y los inherentes al acoplamiento del alumno-institución, del primero, Bean (1980) argumenta que un estudiante toma la decisión de desertar influenciado por factores ajenos a la institución.

Los estudios de Montoya (1999), refieren que los estudiantes con menores ingresos al momento de iniciar sus estudios tienen mayores probabilidades de desertar, fortalece la idea que la deserción considera variables socioeconómicas y el desempeño académico. Driscoll (2006), enlista los factores más comunes que se presentan en las personas que desertan y son: carentes de éxito académico, ausentismo, problemas personales, familiares y económicos.

En otro estudio Bridgeland, Dilulio, y Morison (2006), señalan causas tales como bajos ingresos, pertenecer a minorías, residir en áreas urbanas, familias de un sólo padre, escuelas públicas en zonas empobrecidas. Por otro lado, Tinto (1987) sostiene que la deserción está en función de una falta de integración del alumno con la comunidad universitaria, también menciona que los estudiantes permanecen en la escuela si ellos adquieren identidad con la institución, es decir, que tanto los estudiantes se sienten cómodos en la universidad (Tinto, 1993).

Estudios similares consideran que la deserción está influenciada por: carencia de tiempo, escasa tutoría, poca información sobre el proceso de enseñanza-aprendizaje, falta de soporte y dificultad de comunicación con las instituciones (Fozdar, Kumar y Kankan, 2006), por su parte, Wietse et al., (2011: 34) citan a Raush y Hamilton (2006) quienes ilustran diciendo que "los factores que encontraron fueron la dificultad para socializar y adaptarse al ambiente universitario, la sensación de aislamiento social y académico, así como la apreciación de una falta de integración”, confirmando con esto lo expuesto por Tinto.

De acuerdo con Wietse et al., (2011) cuando citan (ANUIES, 2001), mencionan que en México, el bajo promedio en el bachillerato, el estado civil y la necesidad de los estudiantes en combinar 
los estudios con la actividad laboral, eran los principales factores que intervenían en el sistema educativo en los años ochenta. Por su parte Carrillo Flores, (1993) en sus estudios realizados desde la Universidad de Guadalajara, señala factores tales como el interés por la carrera, el tiempo dedicado al estudio, los métodos, estudio, trabajo simultáneo y el bajo promedio en el bachillerato; elementos que influyen en la decisión de abandono escolar de un alumno.

En este contexto mexicano, Chaín (1995) considera que los factores socioeconómicos tienen un rol importante en la deserción a través de dos enfoques, el primero refiere a los ingresos, tipo de vivienda y la composición familiar, los segundos están influenciados por la escolaridad de los padres, la valoración de la educación, los patrones lingüísticos, los hábitos de estudio y esparcimiento, este último relacionado con la cultura.

Recientemente en la Universidad Autónoma Metropolitana (UAM), Rodríguez J. y Leyva M. (2007: 142) sugieren que el problema de la deserción estudiantil responde a elementos "socioeconómicos y también a factores del desarrollo del sistema educativo, pero no parecen tener mucha influencia en las explicaciones, aspectos relacionados con la integración del estudiante al sistema universitario".

En la Benemérita Universidad Autónoma de Puebla (BUAP), Wietse et al. (2011: 47), aseveran en su estudio que en la deserción universitaria las "condiciones socio-económicas juegan un papel más importante que el clima organizacional o el acoplamiento entre estudiante y universidad", estos últimos autores y para el caso nacional refuerzan las características socioeconómicas del fenómeno.

Como se mencionó al inicio del documento, el mayor número de investigaciones en torno a la deserción estudiantil se han desarrollado en los niveles de educación básica, bachillerato y licenciatura, sin embargo, existen algunos textos que exponen las posibles causas de la deserción en el nivel posgrado, este es el caso de Cooke, Sims y Peyrefitte (1995), quienes en un estudio realizado en los Estados Unidos, mencionan como principal factor de deserción, la actitud de los alumnos hacia el programa de posgrado emprendido, la mayoría de los estudiantes que culminaron el programa ostentaban el compromiso y la necesidad de logro académico, Lovittdes. B. (1996), atribuye la decisión de desertar de los alumnos de posgrado a situaciones complejas que viven dentro de la institución con profesores, tutores, gestores entre otros.

En el estudio realizado por Mdyogolo E. (2012), en The University of Fort Hare en Sud África, considera que, la deficiencia en investigación académica de los estudiantes y las estrategias incorrectas de tutorías, son los elementos principales de la deserción en los alumnos de posgrado. En Argentina, Haro y Valverde (2002), suponen que la deserción en el posgrado se presenta por la cantidad de trabajo y tiempo que requiere invertir el estudiante y que está ligado con el método de enseñanza expositivo. En Venezuela, Dubs R. (2005) concluye que la integración social y académica, relacionados con las competencias del alumno al iniciar los estudios, influyen significativamente en la decisión de permanecer o no en el programa de estudio. 
En Costa Rica, Barrientos Z., y Umaña R. (2009: 146), aluden las causas de deserción en tres sentidos, la primera a la "capacidad docente de los profesores, al grado de concordancia entre el perfil de salida profesional y el mercado laboral y al grado de relación entre el trabajo y el programa académico".

Así López (2009: 884) "recomienda implementar políticas sociales en materia laboral debido a que, por una parte, se percibe que los jóvenes se incorporan a una edad más temprana al trabajo, truncando la continuidad de sus estudios en muchos casos".

En un sentido un tanto alejado al contexto pero interesante de cualquier forma, es la investigación realizada por Vergidis D. y Panagiotakopoulos C. (2002) en The Hellenic Open University, ellos suponen que la deserción en el caso estudiado se presenta por la complejidad de combinar las obligaciones laborales, académicas y familiares, en donde los alumnos subestiman el esfuerzo que requiere el aprendizaje de dicho nivel escolar y el tiempo de dedicación para este. 
Como se puede observar la teoría de la deserción se enfoca principalmente al pregrado y grado, pero también pueden ser aplicados a posgrados universitarios (Himmel 2002). Sintetizando lo expuesto, se puede considerar que los principales factores que influyen en el abandono escolar en nivel licenciatura y posiblemente en posgrado pueden ser clasificados de la siguiente forma:

\section{Esquema 1. Categorías generales de las causas de la deserción}

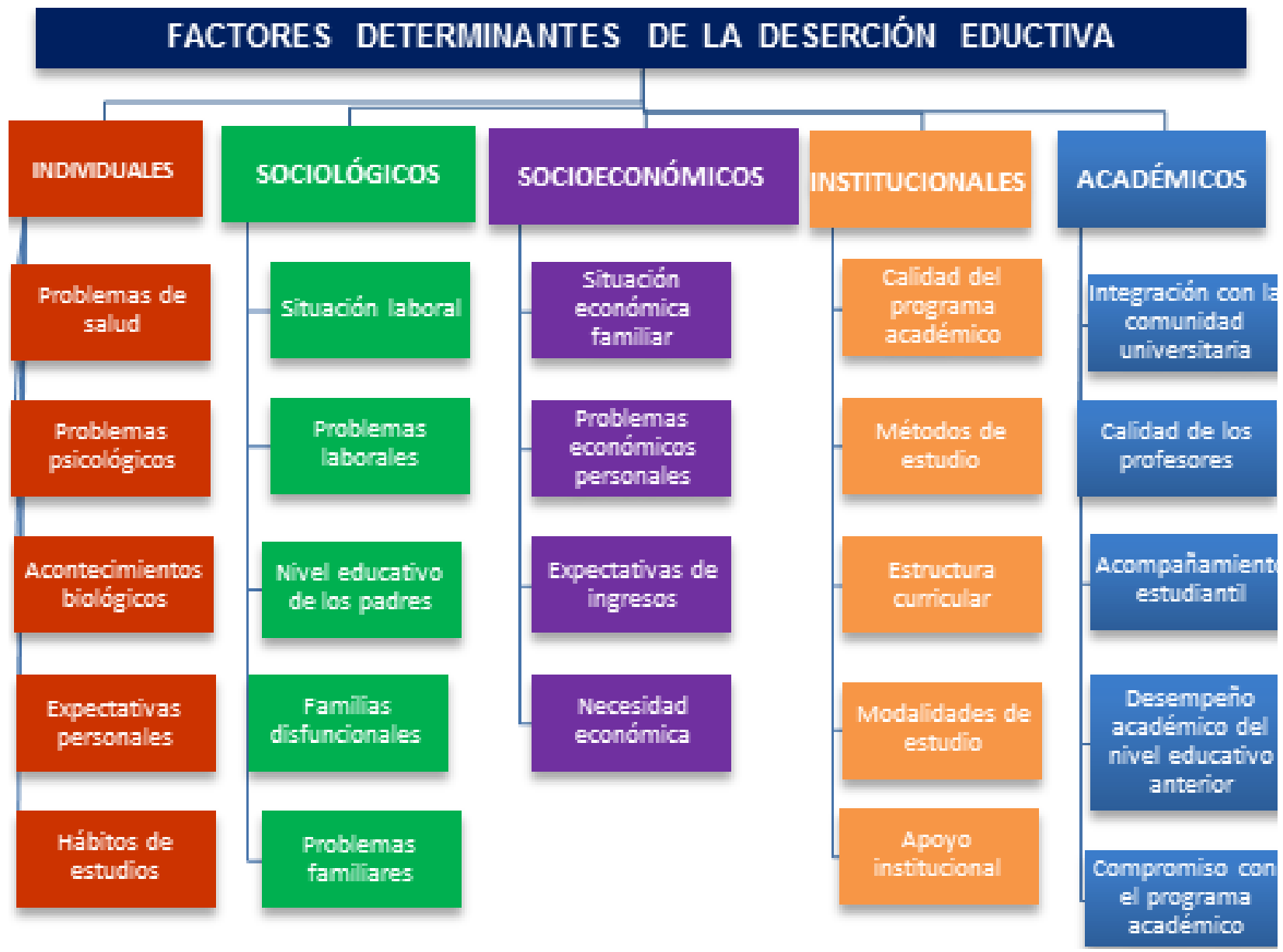

Fuente: elaboración propia con base a los autores citados

Si bien la mayoría de los autores que estudian la deserción en la educación superior a nivel nacional e internacional mencionan múltiples factores que pueden estar involucrados en el abandono escolar, es importante considerar que existe "un peligro, en el terreno de políticas, es presuponer que la deserción obedece a un sinnúmero de factores, (...). Otro peligro es especular, con poca evidencia, que el problema se debe básicamente a un solo factor" Wietse et al., (2011: 46). 
Por tal motivo es importante identificar las características de la deserción para cada contexto, ya que las estrategias de retención deben enfocarse a las variables de cada caso y más para el presente estudio que pretende identificar dichos factores de abandono estudiantil a nivel posgrado, fenómeno poco investigado. Dichas estrategias según Tinto (2006) pueden orientarse en expectativas, consejos, apoyo, acompañamiento y aprendizaje.

\section{Estudio de caso: deserción en los posgrados del Centro Universitario de Ciencias Económico Administrativas (CUCEA)}

La historia de los posgrados CUCEA se remonta hacia el año 1983 con la creación de la Maestría en Administración, que en aquel entonces pertenecía a la Escuela de Graduados, en las calles de Eliseo y Juan Álvarez en la colonia centro de Guadalajara y que formaba parte del departamento de Estudios Regionales. Para 1990 se fundó la Maestría en Economía que pertenecía a la Dirección de Planeación de Evaluación y Desarrollo (DIRPLED), a ésta le siguió la Especialidad de Planeación de la Educación Superior (Posgrados CUCEA, 2006: 10).

En 1990 se forma la Secretaría General Académica con la que aparecen las coordinaciones de investigación y posgrado por cada área de enseñanza. El licenciado Raúl Padilla López, rector General de la Universidad de Guadalajara, establece en 1992 lo que ahora conocemos como la Red Universitaria y se dividen las áreas de aprendizaje dando paso a la creación de los centros universitarios como el CUCEA.

Con esta separación se dio paso a la creación de las distintas dependencias en cada centro universitario y que eran hasta cierto punto un reflejo de la renovada Administración General; como resultado de lo anterior, surge la Coordinación de Posgrado en el CUCEA. Para entonces se integraron ya las maestrías en Economía, Administración, Finanzas e Impuestos y las especialidades en Impuestos y Finanzas (Posgrados CUCEA, 2006, p. 10). Para el año 1996 con todos los avances mencionados, se indujo el nacimiento de más programas de posgrados, mismos que se muestran en la tabla 1.

Tabla 1. Situación de los posgrados en el CUCEA al 2002.

\begin{tabular}{|c|l|}
\hline AÑO & \multicolumn{1}{c|}{ MAESTRÍA } \\
\hline 1998 & $\begin{array}{l}\text { Maestría en Comercio y Mercados Internacionales, Maestría en Auditoría y Maestría en Gestión } \\
\text { Pública. }\end{array}$ \\
\hline 1999 & Maestría en Administración modalidad semi-escolarizada, proveniente del programa Innova. \\
\hline 2000 & Maestría en Mercadotecnia y Maestría en Dirección Estratégica de la Calidad. \\
\hline 2001 & Maestría en Tecnologías para el Aprendizaje y Maestría en Tecnologías de Información. \\
\hline 2002 & Maestría en Gestión y Políticas de la Educación Superior. \\
\hline
\end{tabular}

En el 2003 se da inicio a la reforma de posgrado en la Universidad de Guadalajara, en donde fueron suprimidos y creados nuevos programas de posgrado desde una perspectiva de pertinencia y con visión de futuro, a raíz de esto y a la fecha el CUCEA dispone de 16 programas académicos, 12 Maes- 
trías y cuatro Doctorados, en áreas administrativas, económicas, ciencias políticas, contables, financieras, fiscales, negocios, sistemas de información y educación, en modalidades profesionalizantes y de investigación, de los cuales 12 de ellos tienen reconocimiento del Padrón Nacional de Posgrados de Calidad (PNPC-CONACYT), nueve maestrías y tres doctorados.

Los posgrados CUCEA, son un referente en magnitud y calidad (PNPC) en la Red Universitaria, ya que representan el 12 por ciento de la oferta educativa en dicho nivel ${ }^{1}$, igualmente, atienden a 976 alumnos, equivalentes al 25 por ciento del total de estudiantes matriculados en la universidad, entre los programas de posgrado que desatacan por ofrecer servicios educativos a gran parte de la demanda que tiene el CUCEA son: la Maestría en Administración de Negocios, la Maestría en Dirección de Mercadotecnia y la Maestría en Finanzas Empresariales, las cuales concentran el 43 por ciento de la matrícula.

Respecto a la población estudiantil de los posgrados CUCEA, el género femenino representa el 43 por ciento y el masculino el restante 57 por ciento de la matrícula total y están inscritos en la modalidad profesionalizante (escolarizada y ejecutiva) el 59 por ciento de ellos y de investigación el 41 por ciento restante (tiempo completo-CONACYT). De las 12 maestrías y cuatro doctorados del CUCEA, se identificaron 47 estudiantes que desertaron de 11 programas en los calendarios escolares 2012-A y 2012-B, mismos que representan el 4.8 por ciento del total de la matrícula reportada y que abandonaron sus estudios de los posgrados.

Tabla 2. Relación de deserción estudiantil en los posgrados CUCEA

\begin{tabular}{|c|c|c|c|}
\hline No. & Programa & $\begin{array}{c}\text { Cantidad de desertores } \\
\text { 2012-A }\end{array}$ & $\begin{array}{c}\text { Cantidad de desertores } \\
\text { 2012-B }\end{array}$ \\
\hline 1 & Doctorado en Gestión de la Educación Superior & 1 & $/$ \\
\hline 2 & Maestría en Administración de Negocios & 13 & 2 \\
\hline 3 & Maestría en Finanzas Empresariales & 7 & 2 \\
\hline 4 & Maestría en Análisis Tributario & 1 & 1 \\
\hline 5 & Maestría en Relaciones Económicas & 1 & 1 \\
\hline 6 & Maestría en Negocios y Estudios Económicos & 1 & $/$ \\
\hline 7 & Maestría en Tecnologías del Aprendizaje & 2 & 1 \\
\hline 8 & Maestría en Tecnologías de la Información & 1 & 1 \\
\hline 9 & Maestría en Dirección de Mercadotecnia & 5 & 1 \\
\hline 10 & Doctorado en Estudios Fiscales & $/$ & 15 \\
\hline \hline 11 & Doctorado en Ciencias Económico Administrativas & $/$ & 32 \\
\hline \hline
\end{tabular}

Respecto a los 47 ex alumnos que decidieron interrumpir sus estudios de maestría o doctorado, se obtuvo respuesta de 32 sujetos caso de estudio, lo que significa 68 por ciento del total de desertores. La encuesta utilizada, se estructuró con base a la revisión del estado de arte, y se presentó en cuatro

1 Se consideran únicamente los programas de maestría y doctorado. 
categorías, siendo estas: 1. datos generales., 2. trayectoria escolar., 3. de la institución y 4. opinión, la cual estuvo integrada por 27 reactivos, 13 diseñadas en escala tipo Likert, cinco dicotómicas y siete preguntas abiertas y fue aplicada vía correo electrónico.

\section{Análisis de los resultados}

Respecto a los resultados obtenidos en el apartado de datos generales, se observa que del total de los encuestados 60 por ciento de los ex alumnos que desertaron de los posgrados tienen una edad menor a 25 y 30 años, 33 por ciento entre 31 y 35 años, sólo 7 por ciento tenía una edad mayor a 36 años; en este sentido el 50 por ciento de los entrevistados son damas y el restante caballeros, de estos 72 por ciento son solteros y 28 por ciento casados, la mayoría de ellos, 78 por ciento proceden del Estado de Jalisco, el otro 19 por ciento está dividido entre Yucatán, Baja California, el Distrito Federal, Mérida, Puebla y Chiapas, respecto a su estatus escolar 20 por ciento de los desertores eran becados por CONACYT y el restante 80 no recibieron apoyos económicos.

Con relación a su actividad laboral 100 por ciento disponen de un empleo actualmente y de 1 a 7 años de antigüedad, respecto a su ingreso mensual 50 por ciento de los desertores escolares perciben entre menos de $\$ 5000$ y $\$ 10000$ pesos, el 25 por ciento entre $\$ 11000$ y $\$ 15000,6$ por ciento gana entre $\$ 16000$ y $\$ 20000$, en ese mismo porcentaje (6) reportaron ingresos hasta $\$ 30000$ y el 13 por ciento de los consultados tiene ingresos mayores a \$35 000 pesos (véase gráfica 1).

\section{Gráfica 1. Nivel de ingreso de los ex alumnos (representado en miles)}

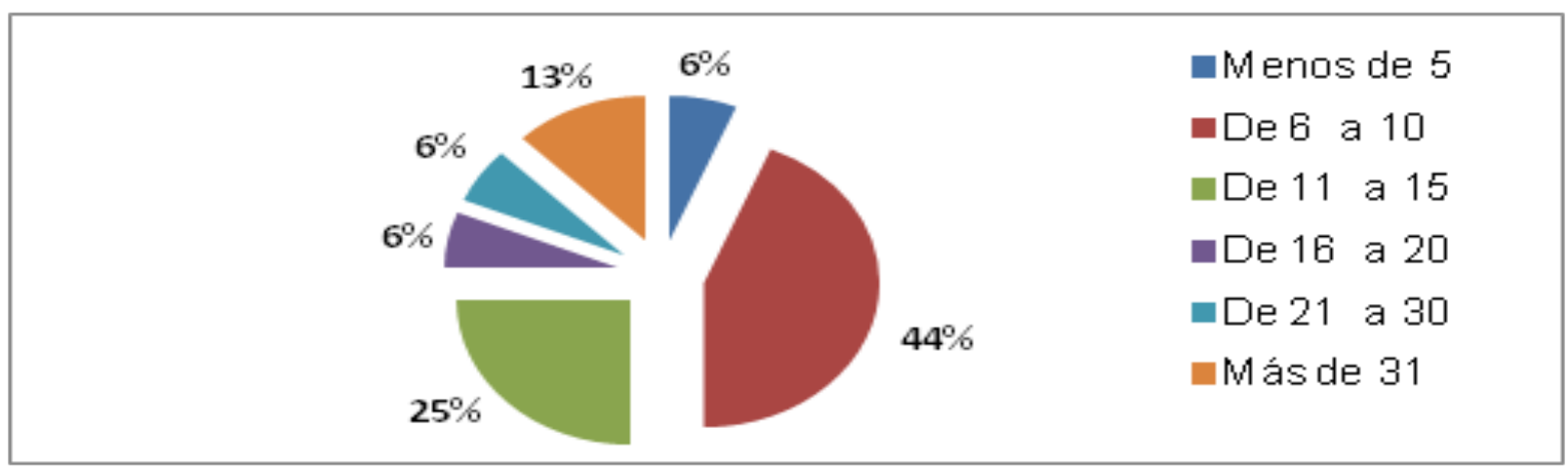

Fuente: Elaboración propia con datos de la encuesta a desertores.

Con relación a la institución en que laboran 69 por ciento de los ex alumnos mencionan colaborar en empresas grandes, 19 por ciento en pequeñas, 6 por ciento en medianas y seis en micro, de estas empresas la mayoría 37 por ciento trabaja en instituciones educativas, 29 por ciento en la industria de manufactura y transformación, 19 por ciento en compañías comerciales como las de mayor re presentación (véase gráfica 2). 
Gráfica 2. Tipo de empresa en dondeaboran los ex alumnos

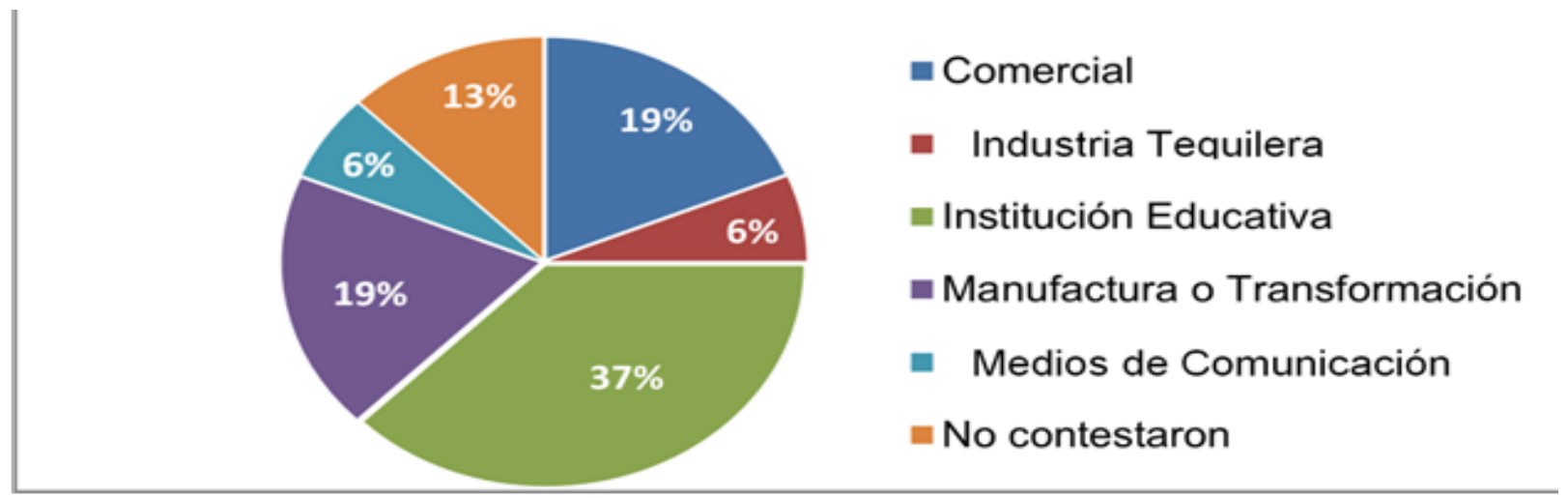

Fuente: Elaboración propia con datos de la encuesta a desertores.

De acuerdo al ambiente familiar 60 por ciento mencionó no tener dependientes económicos, el restante 40 por ciento son responsables de 1 a 4 personas, en este sentido la escolaridad que tiene la madre de los encuestados es de licenciatura en 35 por ciento, de bachillerato en 21 por ciento y de primaria con 19 por ciento, para la educación secundaria es 12 por ciento, con 13 por ciento las madres que tienen un posgrado (véase gráfica 3).

Gráfica 3. Último grado de estudios de las madres de los ex alumnos

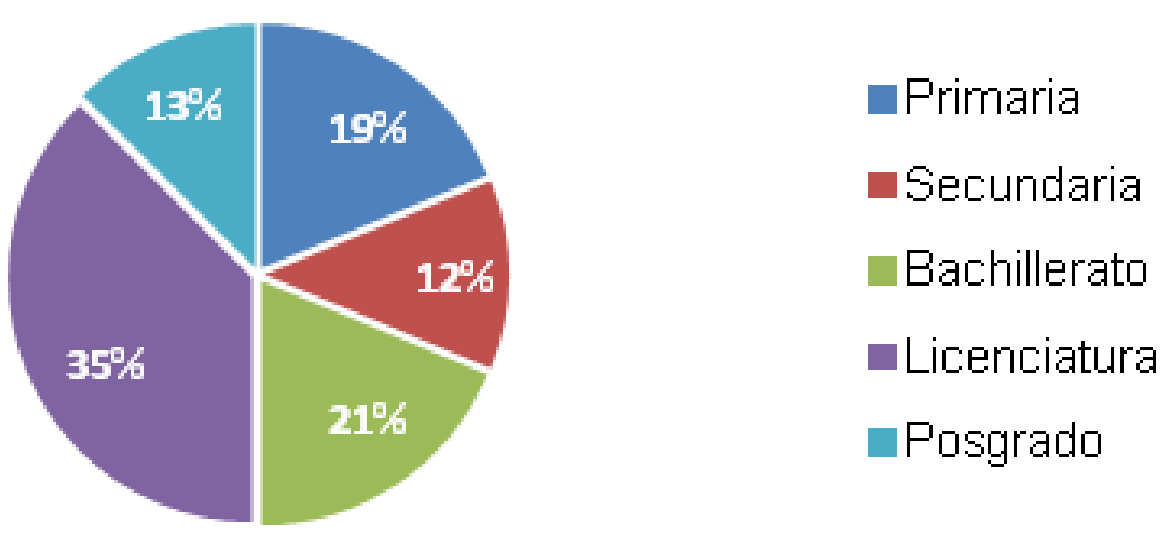

Fuente: Elaboración propia con datos de la encuesta a desertores. 
Para el caso de los padres 52 por ciento tienen licenciatura y con 13 por ciento se encuentran los estudios de posgrado y bachillerato, con 10 por ciento secundaria y 12 por ciento para los padres con estudios de primaria. (Véase gráfica 4)

\section{Gráfica 4. Último grado de estudios de los padres de los ex alumnos}

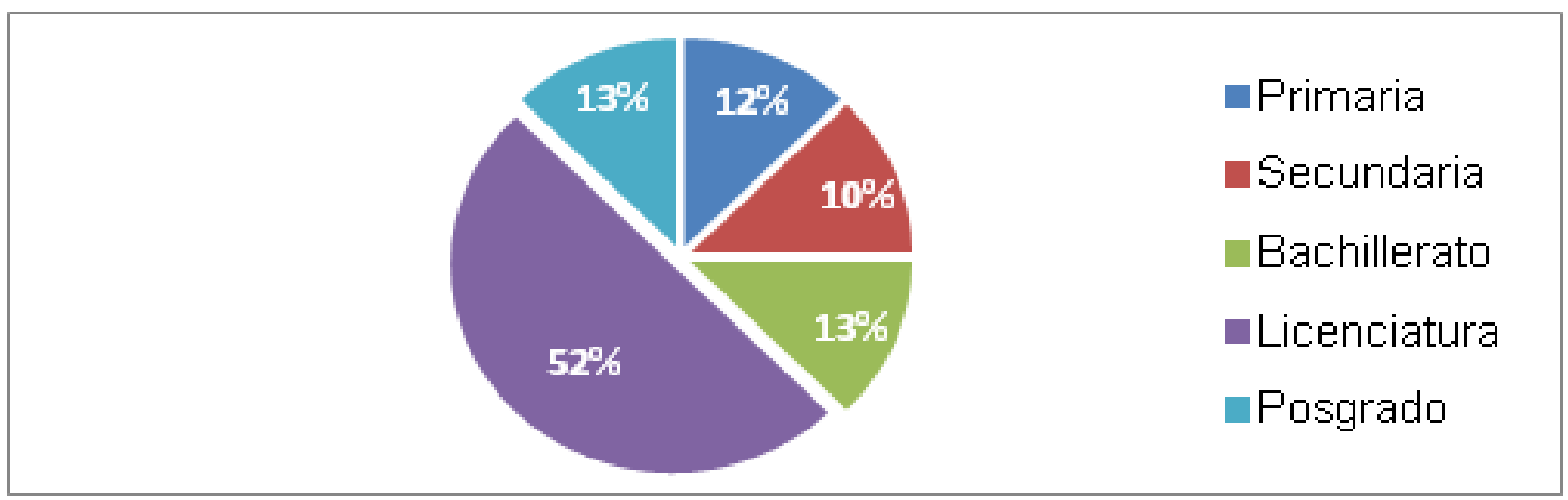

Fuente: Elaboración propia con datos de la encuesta a desertores.

Del apartado de antecedentes y trayectoria escolar 80 por ciento de los ex alumnos que interrumpieron sus estudios provienen de instituciones educativas públicas el restante 20 por ciento de privadas, con base a la licenciatura de procedencia 30 por ciento estudio ingeniería, 22 por ciento contaduría y mercadotecnia con el mismo porcentaje, y con 8 por ciento nutrición, procedentes de la licenciatura en administración se presentó 15 por ciento, como las principales, de éstos el 10 por ciento de los encuestados mencionó haberse ausentado un periodo escolar durante la licenciatura.

Respecto al promedio de licenciatura el 58 por ciento obtuvo una calificación final de entre 86 a 90 puntos, 22 por ciento logró una nota de 80 a 85, 16 por ciento de 90 a 95 y sólo el cuatro por ciento más de 95 , en promedio las calificaciones de los desertores en su último ciclo escolar en el posgrado fue de 90.48 , respecto al semestre en que desertaron 70 por ciento lo hizo en el primer ciclo escolar, 25 por ciento en el segundo y el 5 por ciento en tercer semestre. De la tercera parte institucional, se preguntó los motivos que tuvieron para estudiar un posgrado en la UdeG., las principales respuestas fueron: 1. por el nivel académico de la institución, 2. por la calidad de sus posgrados (CONACYT), 3. por los costos accesibles y 4. por recomendación de egresados y estudiantes activos; igualmente se consultó que tanto se sentía satisfecho con el programa de posgrado que estudiaba a lo cual respondieron algo satisfecho en 50 por ciento y completamente 44 por ciento (véase gráfica 5). 
Gráfica 5. Grado de satisfacción de los ex alumnos con el programa de estudio del posgrado

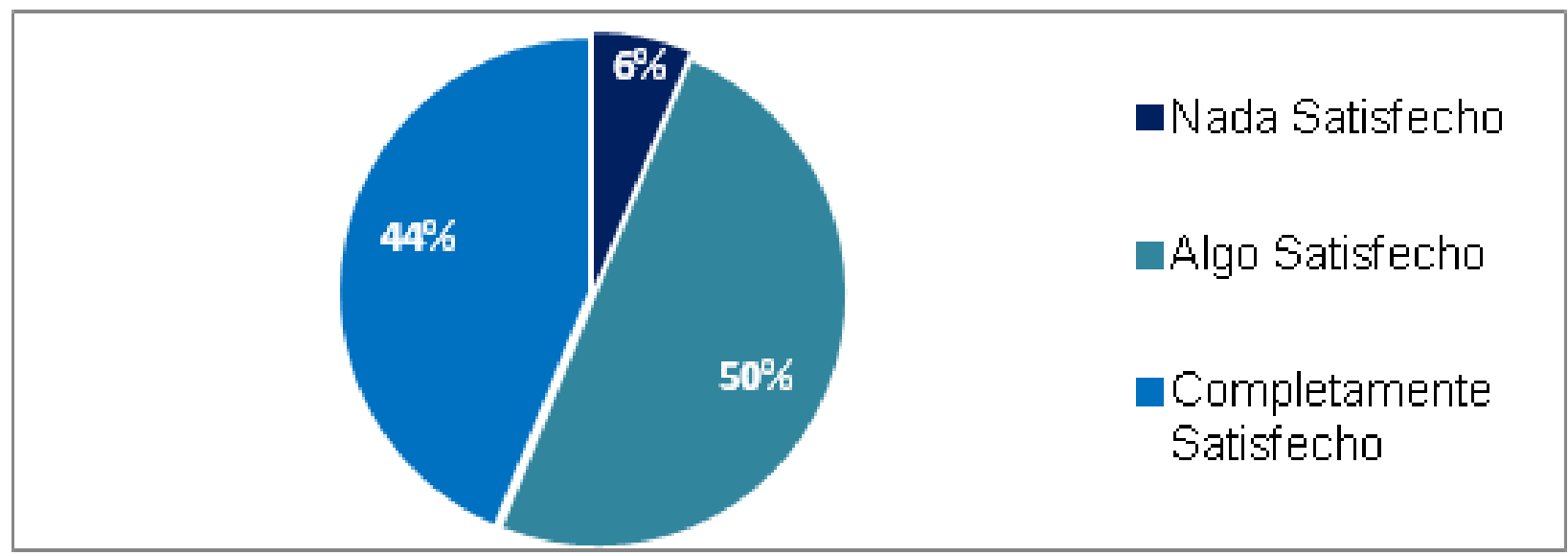

Fuente: Elaboración propia con datos de la encuesta a desertores.

Respecto a su relación con los compañeros de grupo, mencionan que era excelente en 70 por ciento, buena en 26 por ciento y el restante regular, por otro lado se preguntó su percepción respecto al apoyo institucional para su permanencia en el posgrado a lo que opinaron 56 por ciento que recibieron el apoyo suficiente, 38 por ciento algún apoyo y el restante indicó que no hubo apoyo (véase gráfica 6).

Gráfica 6. Apoyo recibido de parte de la universidad para continuar con los estudios

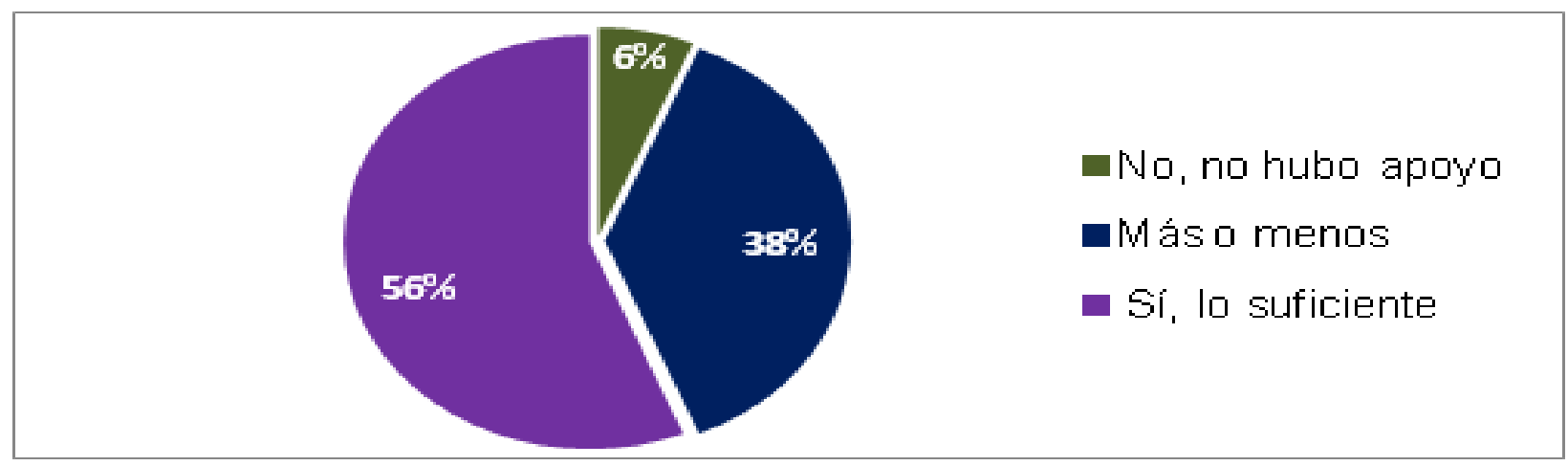

Fuente: Elaboración propia con datos de la encuesta a desertores. 
Finalmente del cuarto aparatado de opinión se preguntó quién decidido que debía interrumpir los estudios de posgrado, las respuestas expresadas fueron: 76 por ciento el propio ex alumno, y con 8 por ciento los padres, pareja y otros (véase gráfica 7).

\section{Gráfica 7. ¿Quién decidió dar por terminado el posgrado?}

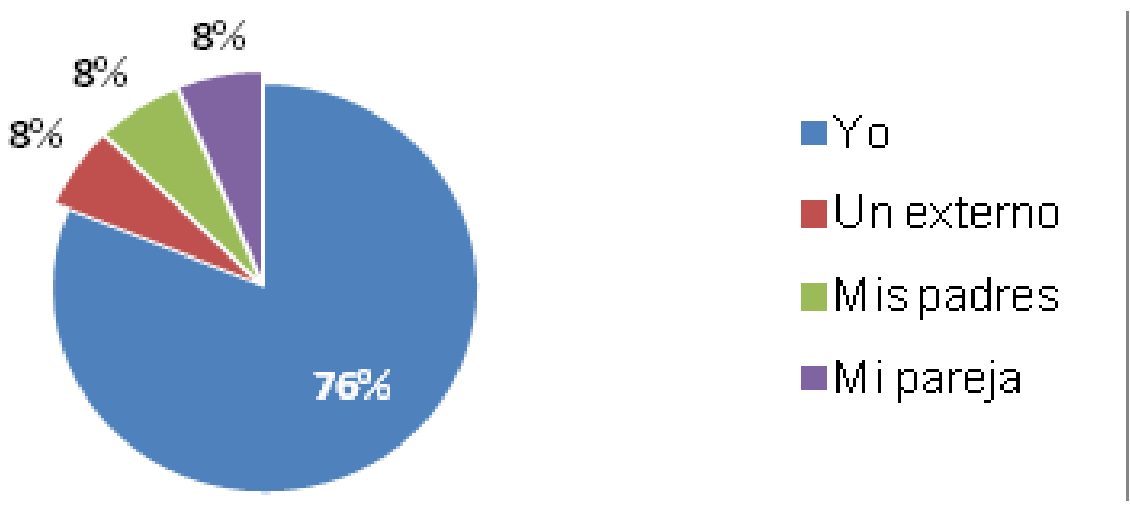

Fuente: Elaboración propia con datos de la encuesta a desertores.

Por otro lado se consultó cuáles factores motivaron su decisión de abandonar la universidad, destacando tres:

1. Problemas laborales con 42 por ciento, en el cual se sobresalen las diferencias con los superiores, ubicación de la institución con relación a su lugar de trabajo, el sueldo no era el deseado, el rol del ex alumno en la organización, entre otros;

2. Problemas personales con 24 por ciento, en este punto únicamente se mencionó problemas con la pareja y familiares, y

3. Problemas económicos con 12 por ciento, relacionados a la escasez de dinero y vinculada a la crisis económica nacional (véase gráfica 8).

\section{Gráfica 8. Principales variables que intervienen en la deserción de los posgrados CUCEA}

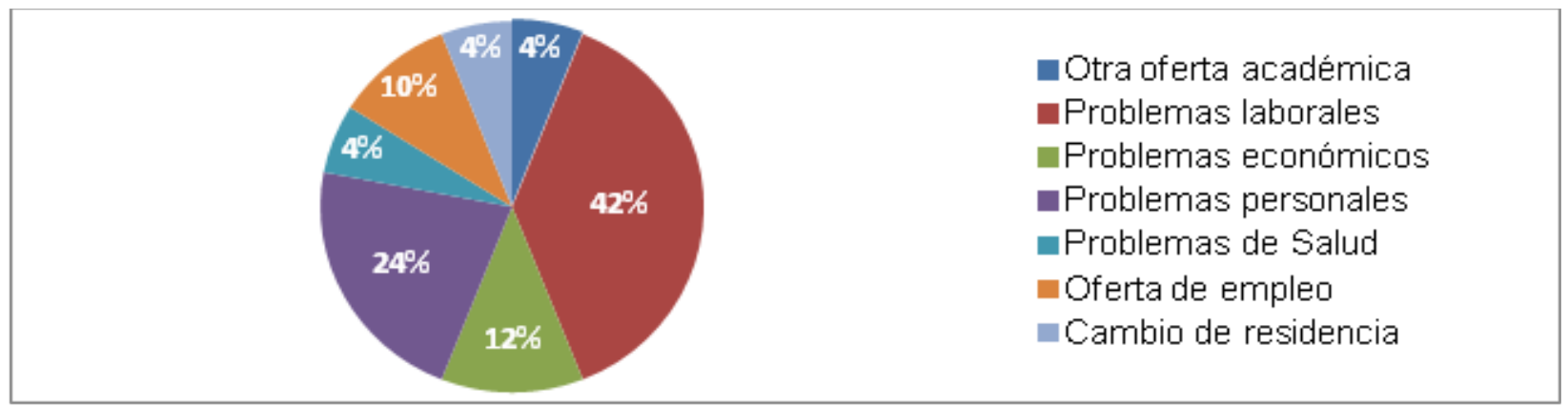

Fuente: Elaboración propia con datos de la encuesta a desertores. 
En este contexto, se consultó cuáles son las actividades que realizan actualmente en las horas que dedicaba a estudiar el posgrado: 1. permanecer más tiempo en el trabajo, 2. dedicar más tiempo a la familia, 3. atender otro trabajo, 4. actividad física (ejercicio) y 5. estudiar, como las principales, por último se preguntó si tenían considerado regresar a sus estudios de posgrado a lo cual respondieron lo siguiente:

\section{Gráfica 9. ¿Considera continuar los estudios de posgrado?}

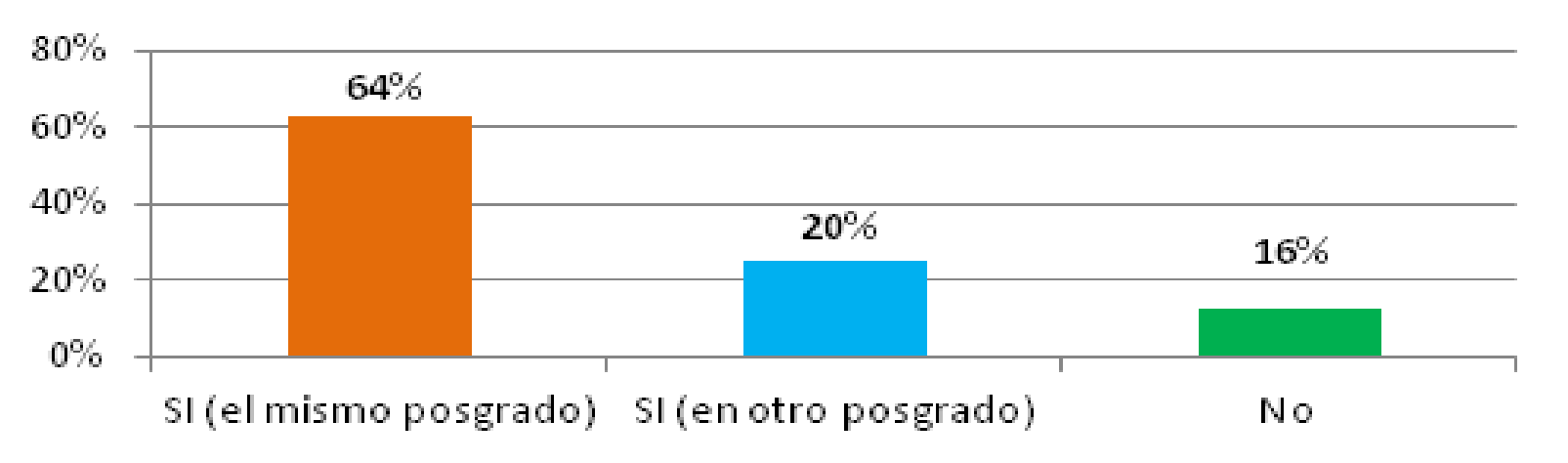

Fuente: Elaboración propia con datos de la encuesta a desertores.

De los resultados obtenidos, podemos identificar un patrón de abandono diferente en detalles a los expuestos en la teoría, considerando el grado académico, las dinámicas laborales y personales de los sujetos de estudio, es normal que los motivos de deserción no sean similares a los casos de nivel pregrado o grado.

\section{Consideraciones finales}

Es importante mencionar que en los posgrados CUCEA, la deserción de los estudiantes está marcada, principalmente, por factores sociológicos y socioeconómicos, en donde estos últimos son menos representativos, aspecto contrario a la situación económica del país; igualmente, no hay una tendencia marcada con aspectos de género, nivel académico de los padres ni con el rendimiento escolar de los ex alumnos antes o durante el estudio del posgrado.

En este sentido, es significativo destacar que no se presentaron factores institucionales o académicos que motivaran el abandono de las aulas, pero si se hace necesario considerar estrategias en materia curricular para mantener a los alumnos en la universidad, ya que en los aspectos sociológicos mencionados, predominan los de carácter laboral en donde la obligación de asistir a clases presenciales, los horarios y el propio estrés que genera estudiar un posgrado, vinculado con el trabajo y en muchos casos con la responsabilidad familiar, pudieran ser un detonante de presión, en general para su rendimiento laboral y personal, que estaría impactando directa o indirectamente la deserción del posgrado. 
Finalmente, la información proporcionada por los desertores representa un punto de vista muy importante sobre el funcionar y contexto de los programas de posgrado en general, y que debidamente atendidos pueden convertirse en estrategias o realidades que gradualmente mitiguen la deserción y mejoren el desempeño institucional en beneficio de los alumnos y la eficiencia terminal. Puntualmente se recomienda formular estrategias más robustas en los procesos de ingreso, en el acompañamiento escolar y en la flexibilidad del currículo con el apoyo de las tecnologías de información y comunicación o mediante la integración de cursos en modalidades no convencionales.

\section{Bibliografía}

Arboleda, P. y C. Picón (1997). La Mortalidad y la deserción estudiantil en EAFIT, y sus causas y posibles soluciones. Medellín, Colombia: Dirección de Planeación EAFIT.

Barrientos, Z. y R. Umaña (2009). "Deserción estudiantil en posgrados semi presenciales de la Universidad Estatal a Distancia (UNED)”. Costa Rica: ¿Deserción o retraso?, Cuadernos de Investigación UNED (Edición en Línea ISSN: 1659-4266) 1(2), Costa Rica.

Bean, J. (1980). "Dropouts and Turnover: The Synthesis and Test of a Casual Model of Student Attrition”. Research in Higher Education, 12, pp. 155-187.

Bridgeland, J. M., J. J. DiIulio, K. B. Morison (2006).The Silent Epidemic: Perspectives of High School Dropouts. Seattle: Bill \& Melinda Gates Foundation.

Carrillo, I. (1993). "El abandono escolar en educación superior; ¿una decisión racional o un efecto multifactorial involuntario?". Eficiencia Terminal y Calidad Académica en las IES. México: Universidad de Guadalajara.

Chaín, R. (1995). Estudiantes Universitarios: Trayectorias escolares, Universidad Veracruzana. Universidad Autónoma de Aguascalientes.

Cooke, D., R. Sims y J. Peyrefitte (1995). "The Relationship Between Graduate Student Attitudes and Attrition". The Journal of Psychology: Interdisciplinary and Applied 129 (6).

Driscoll, D. P. (2006). Dropouts in Massachusetts Public Schools: District Survey Results. Malden: Massachusetts Department of Education.

Dubs, R. (2005). Permanecer o desertar en los estudios de postgrado: Síntesis de modelos teóricos. Investigación y Postgrado, Recuperado el 20 de junio de 2013, desde: http://www.scielo. org.ve.

Fozdar, B., L. Kumar y S. Kannan (2006). "Study of the Factors Responsible for the Dropouts from the BSC Programme of Indira Gandhi National Open University". International Review of Research in Open and Distance Learning, 3, pp. 1492-3831.

Hackman, J. y W. S. Dysinger (1970). "Commitment to College as a Factor in Student Attrition". Sociology of Education, 43, pp. 311-324. 
Haro M. y M. Valverde (2002). "New estrategies for teaching immunology in the school of medicine and in the postgraduate course. Relationships between methodologic preferences with evaluation results and desertion index". Cátedra de Histología, Facultad de Medicina, U.N.T $33(1)$.

Himmel, E. (2002). "Modelos de análisis para la deserción estudiantil en la educación superior". Calidad de la Educación: Retención y movilidad estudiantil en la educación superior, 17, 91107. Recuperado el 5 de mayo de 2013, desde:

http://www.cned.cl/public/secciones/seccionrevistacalidad/revista_calidad_leer_revista. aspx?idPublicacion $=35$

Kember, D. (1989). A Longitudinal-process Model of Dropout From Distance Education. Journal of Higher Education, 60, pp. 278-301.

López, M. (2009). "Efectos de la correspondencia entre educación y empleo de los jóvenes en el mercado de trabajo mexicano". RMIE, 14 (42), pp. 863-887. España.

Lovittdes, B. (1996). "Who Is Responsible for Graduate Student Attrition The Individual or the Institution? Toward an Explanation of the High and Persistent Rate of Attrition". Education Resources Information Center.

Magallón L., C. Calderón y E. Soto (2006). Estudio de la deserción estudiantil de los estudios de pre grado de la Universidad de los Llanos. Apial, Colombia: Universidad de los Llanos.

Mdyogolo, E. (2012). The academic experiences of Faculty of Education postgraduate students who have dropped out of a Higher Education Institution in Eastern Cape Province. Eastern Cape, South Africa: The University of Fort Hare.

Montoya, M. D. (1999). "Extended stay at university: an application of multinomial logit and duration models". Applied Economics, 31, pp. 1411-1422.

Muñoz, I., P. Rodríguez, P. Restrepo y C. Borrani (1979). "El síndrome del atraso escolar y el abandono del sistema educativo". Revista Latinoamericana de Estudios Educativos. 9 (3).

Organización para la Cooperación y el Desarrollo Económico (OCDE) (2004). Problem Solving for Tomorrow's. Paris: OECD Publications.

Osorio, A. y C. Jaramillo (1999). Deserción estudiantil en los Programas de Pregrado 1995-1998. Medellín: Documento.

Páramo, G. y C. Correa (1999). "Deserción estudiantil universitaria. Conceptualización”. Revista Universidad Eafit. Colombia. Abril-Mayo-Junio, pp. 65-78.

Posgrados CUCEA (2006). Tu futuro asegurado. México: Universidad de Guadalajara.

Rodríguez, J. y M. Leyva (2007). “La deserción escolar universitaria. La experiencia de la UAM. Entre el déficit de la oferta educativa superior y las dificultades de la retención escolar”. Revista REDALYC, 22 (14298-111).

Rumberger, R. W. (2001). Why students drop out of school and what can be done. Civil Rights Project, Harvard University. 
Secretaría de Educación Pública (SEP) (2004). Estadísticas básicas del sistema educativo del estado de Sonora. Inicio de cursos 2003-2004. Hermosillo: Gobierno del Estado de Sonora.

Tinto, V. (1987). "El abandono de los estudios superiores: Una nueva perspectiva de las causas del abandono y su tratamiento". Cuaderno de Planeación Universitaria. Año 6 (2), octubre 1992. México: UNAM, ANUIES.

(1993). Leaving college: rethinking the causes and cures of student attrition. Chicago: University of Chicago Press.

- (2006). "Research and practice of student retention: what is next?". Journal of college student retention, 8 (1) pp. 1-19.

Vergidis, D. y C. Panagiotakopoulos (2002). "Student Dropout at the Hellenic Open University: Evaluation of the graduate program, Studies in Education". The international review of research in open and distance learning.

Wietse, V., P. León, J. Romero y I. Hernández (2011). “¿Desertores o decepcionados? Distintas causas para abandonar los estudios universitarios". Revista de la educación superior, XL (4), No. 160, México.

Zúñiga, M. (2006). Deserción Estudiantil en el Nivel medio Superior. Causas y solución. México: Trillas.

Recibido: 28/09/13

Dictaminado: $12 / 03 / 14$

Corregido: 24/03/14

Aceptado: 03/04/14 